\title{
Oral Calcium Supplements Do Not Affect the Progression of Aortic Valve Calcification or Coronary Artery Calcification
}

\author{
Mayurkumar Bhakta, MD, Charles Bruce, MD, David Messika-Zeitoun, MD, \\ Lawrence Bielak, DDS, Patrick F. Sheedy, MD, Patricia Peyser, PhD, and \\ Maurice Sarano, MD
}

Background: The use of oral calcium supplementation among the elderly for prevention and treatment of osteoporosis and osteopenia is increasing. The incidence of aortic valve disease and coronary artery disease also is increasing. No study thus far has been done to demonstrate whether this affects the progression of calcification in both the valves and vasculature. We sought to determine whether ingestion of oral calcium supplementation has an effect on aortic valve calcification (AVC) and coronary artery calcification (CAC).

Methods: We performed an independent assessment of AVC, CAC, and calcium supplementation among patients enrolled in the Epidemiology of Coronary Artery Calcification study who were $>60$ years of age and had baseline and 4-year follow-up AVC data. In this population-based study of Olmsted County (Minnesota) residents, AVC and CAC scores were determined prospectively by electron beam computed tomography. We evaluated baseline demographic data and analyzed whether those patients using calcium supplementation had a higher rate of progression of both AVC and CAC.

Results: We identified 257 patients (mean age, $67.8 \pm 5.2$ years), 144 of whom were women. Twenty-five patients (all women) reported using calcium supplements. Analysis of the 144 women (25 taking calcium supplementation) showed there was no difference in the progression of AVC (mean difference in baseline and follow-up AVC score; no supplement versus supplement, $30 \pm 9$ vs $39 \pm 28 ; P=.73$ ) or CAC (mean difference in baseline and follow-up CAC score; no supplement vs supplement, $47 \pm 15$ vs $112 \pm 22 ; P=.154)$. There were no significant differences between the 2 groups with regard to baseline AVC, serum calcium, renal function, diabetes, hypertension, cholesterol, or body mass index.

Conclusion: In this community-based observational study with a 4-year follow-up, no significant increased progression of AVC or CAC was found in women taking oral calcium supplementation. Larger prospective, randomized studies are needed to confirm these findings. (J Am Board Fam Med 2009;22: 610-616.)

The aging population of the United States has been accompanied by a concomitant increase in agerelated disorders. Two such entities include postmenopausal osteoporosis and degenerative aortic valve stenosis caused by progressive aortic valve

This article was externally peer reviewed.

Submitted 17 October 2008; revised 23 February 2009; accepted 2 March 2009.

From the Mayo Clinic, Scottsdale, AZ (MB) and Rochester, MN (CB, PFS, MS); Bichat Hospital, Paris, France (DM-Z); and the University of Michigan, Ann Arbor (LB, PP).

Funding: none.

Conflict of interest: none declared.

Corresponding author: Mayurkumar Bhakta, MD, The Mayo Clinic Arizona, 13400 E. Shea Blvd, Scottsdale, AZ 85259 (E-mail: bhakta.mayurkumar@mayo.edu). calcification (AVC), ${ }^{1}$ or Mönckeberg disease. Osteoporosis is a condition that is characterized by a loss of skeletal bone mass and a concomitant increase in fracture rate. Guidelines from the American Association of Endocrinologists, ${ }^{2}$ the National Osteoporosis Foundation, ${ }^{3}$ and the US Surgeon General ${ }^{4}$ recommend oral calcium supplementation as a measure of prevention and treatment. These guidelines have led to a rise in the use of calcium supplementation among the general population.

Aortic valve stenosis from AVC is the most frequent valve disease encountered in clinical practice $^{5}$ and represents a major public health burden. ${ }^{6}$ Recent data has shown that AVC and subsequent 
stenosis is an active process. ${ }^{7}$ Multiple factors, such as endothelial disease, ${ }^{8}$ inflammation, ${ }^{9}$ and atherosclerosis, ${ }^{10}$ have been implicated as contributors to the formation and progression of AVC. It has also been suggested that calcific valvular and vascular disease have similarities to bone remodeling, ${ }^{11}$ raising concern that supplemental calcium taken to increase or maintain bone density may also increase AVC and vascular calcification. This concern has neither been supported nor alleviated by appropriate measurement of AVC or coronary artery calcification (CAC) in patients taking calcium supplements.

Thus, to investigate whether ingestion of oral calcium supplements affects the severity and progression of AVC and CAC, we took advantage of the prospective Epidemiology of Coronary Artery Calcification (ECAC) study in which AVC and CAC was objectively scored by high-resolution computed tomography. We identified those patients who reported taking calcium supplementation and compared their rates of AVC and CAC to the remainder of the cohort who did not use calcium supplements. ${ }^{7}$

\section{Methods}

\section{Study Population}

This study was reviewed and approved by The Mayo Clinic Institutional Review Board. The database of the AVC substudy of the ECAC provided the patient population for our study. ${ }^{12}$ The ECAC database consists of 1376 randomly selected adult Olmsted County (Minnesota) residents of all ages. After obtaining informed written consent, these patients were prospectively and repeatedly examined by electron beam computed tomography (EBCT) for cardiac calcifications in addition to a comprehensive clinical and cardiac risk factor assessment. Exclusion criteria for ECAC were active pregnancy, lactation at enrollment, or previous cardiac surgery. The AVC substudy further limited enrollment to patients $\geq 60$ years old who had a baseline and follow-up EBCT performed after 1995. Analysis of the effect of calcium supplementation on AVC was performed on the overall population and then limited to women only because no men reported taking calcium supplementation.

\section{Measures and Definitions}

We performed a retrospective review of the clinical charts of all patients enrolled in the ECAC AVC substudy. The chart reviewer was blinded to EBCT scores while compiling a database consisting of patients' calcium supplementation use (irrespective of dose); measured serum creatinine; calculated glomerular filtration rate (GFR); and measured serum calcium at initial evaluation. No data were collected with regard to dietary intake of calcium. Additional variables of interest included age, sex, blood pressure, history of diabetes, tobacco use, lipid profile, myocardial infarction history, physician-diagnosed hypertension, myocardial infarction, and stroke. Hypertension was diagnosed and/or confirmed by physician examination using a Hawksley sphygmomanometer (Lancing, Sussex, England) in the right arm with a random-zero. Three measures were taken, each 2 minutes apart, and the average of the second and third measurements was recorded and used. Patients with a systolic blood pressure $\geq 140 \mathrm{~mm} \mathrm{Hg}$ or diastolic blood pressure $\geq 90 \mathrm{~mm} \mathrm{Hg}$ were considered hypertensive if they did not already have the diagnosis. Patients using insulin or oral hypoglycemic agents were considered diabetic. Body mass index was calculated by dividing the weight $(\mathrm{kg})$ by the square of the height (meters). Standard automated laboratory measures for calcium and creatinine were used. Standard enzymatic methods after overnight fast for total cholesterol, high-density lipoprotein (HDL) cholesterol, and triglycerides were used. Low-density lipoprotein (LDL) was calculated using the Friedwald equation. GFR was approximated using the Modification of Diet in Renal Disease (MDRD) equation ${ }^{13}$ because it takes into account age, sex, and race when calculating approximate GFR.

\section{Electron Beam Computed Tomography}

The images were obtained using a Imatron C-100 or C-150 (San Francisco, CA) triggered at $80 \%$ of RR intervals for 2 chest scan runs ${ }^{14}$ of 30 to 40 contiguous transverses slices (3-mm thickness; $100 \mathrm{~ms} / \mathrm{slice}$ ). Calcification was defined by at least 4 adjacent pixel nodules with density $>130$ Hounsfield units. Dedicated software ${ }^{15}$ was used to calculate Agatston units (AUs) separately for AVCs. Calcium identification on imaging was automated and highlighted all calcifications. Operators then selected which calcifications were on the aortic valve and which were on the aorta or coronary arteries. Two runs were scored separately and averaged. Progression rates of AVC were calculated as the difference between baseline and follow-up 
Table 1. Baseline Characteristics in the Overall Population and Subgroups of Those Who Take and Do Not Take Calcium Supplementation*

\begin{tabular}{|c|c|c|c|c|}
\hline Variable & $\begin{array}{c}\text { Overall } \\
(\mathrm{n}=257)\end{array}$ & $\begin{array}{l}\text { No Supplement } \\
\quad(\mathrm{n}=232)\end{array}$ & $\begin{array}{l}\text { Supplement } \\
(\mathrm{n}=25)\end{array}$ & $P$ \\
\hline Age (yr) & $67.8 \pm 5.2$ & $67.7 \pm 5.1$ & $68.5 \pm 5.9$ & .45 \\
\hline Male Gender (n [\%]) & $113(44)$ & $113(49)$ & $0(0)$ & $<.001$ \\
\hline Body Mass Index $\left(\mathrm{kg} / \mathrm{m}^{2}\right)$ & $28.6 \pm 5.1$ & $28.8 \pm 5.1$ & $27.2 \pm 4.7$ & .13 \\
\hline Age $<70$ years $(\mathrm{n}[\%])$ & $168(65)$ & $154(66)$ & $14(56)$ & .3 \\
\hline Smoking (pack/yr) & $13.7 \pm 21.8$ & $14.5 \pm 22.3$ & $6.5 \pm 14.1$ & .08 \\
\hline History of HTN (n [\%]) & $140(54)$ & $128(55)$ & $12(48)$ & .49 \\
\hline Treated DM (n [\%]) & $25(10)$ & $24(10)$ & $1(4)$ & .31 \\
\hline Tchol (mg/dL) & $209.6 \pm 34.6$ & $207.9 \pm 34.7$ & $225 \pm 30.1$ & .018 \\
\hline LDL (mg/dL) & $121.9 \pm 29.0$ & $121.3 \pm 29.2$ & $127.8 \pm 27.3$ & .28 \\
\hline HDL (mg/dL) & $52.6 \pm 15.7$ & $51.8 \pm 15.7$ & $60.1 \pm 13.3$ & .012 \\
\hline TG (mg/dL) & $176.9 \pm 81.4$ & $175.9 \pm 82.2$ & $185.8 \pm 74.6$ & .57 \\
\hline CAC score (base) & $317.5 \pm 645.4$ & $346.3 \pm 672.6$ & $50.4 \pm 79.3$ & .029 \\
\hline AVC score (base) & $54.5 \pm 174.4$ & $57.7 \pm 181.6$ & $25.4 \pm 77.9$ & .38 \\
\hline Serum calcium & $9.5 \pm 0.3$ & $9.5 \pm 0.3$ & $9.4 \pm 0.3$ & .88 \\
\hline GFR (MDRD) & $67.9 \pm 14.7$ & $67.7 \pm 15.0$ & $69.3 \pm 12.7$ & .62 \\
\hline
\end{tabular}

*Data source: the Epidemiology of Coronary Artery Calcification (ECAC) study. ${ }^{12}$

HTN, hypertension; DM, diabetes mellitus; LDL, low-density lipoprotein; HDL, high-density lipoprotein; TG, triglycerides; CAC, coronary artery calcification; AVC, aortic valve calcification; GFR, glomerular filtration rate; MDRD, Modification of Diet in Renal Disease equation.

score divided by the follow-up duration. To avoid misdiagnosis of change in regard to interrun variability, the progression was defined categorically based on a validated regression approach. The follow-up calcification score was compared with the baseline calcification $95 \%$ CIs, and progression was defined by a score $>95 \%$ CI upper limit, regression by a follow-up score $<95 \% \mathrm{CI}$, and stability by score between the $95 \%$ CI lower and upper limits.

\section{Statistical Analysis}

Statistical analysis was done on JMP software (version 6.0.0; SAS Institute, Inc., Cary, NC). All data presented are in the form of mean $\pm \mathrm{SD}$ or as a percentage. Comparison between groups was done using standard $t$ test, either on regular or log values, or Wilcoxon rank sum test for non-normally distributed variables. Stepwise multiple linear regression analysis helped to determine progression rate from baseline to follow-up.

\section{Results}

\section{Baseline Characteristics}

Of the total number of patients enrolled in the original ECAC database, 257 participants aged 60 years or older had AVC and CAC studies. From this group of 257 patients, we identified those who reported using calcium supplementation (Table 1). We found statistically significant baseline characteristic differences in CAC scores and HDL levels among these patients. Because 113 out of the total 257 participants were men, none of whom were taking supplements, we performed a gender-based analysis, evaluating the effect of calcium supplementation in women only. There were a total of 144 women, 25 of whom reported using calcium supplementation (Table 2). The baseline comparison of the 2 cohorts of women (calcium supplements vs no supplements) showed no significant differences in CAC, AVC, serum calcium levels, GFR, total cholesterol, HDL, LDL, or triglyceride). Only one woman with diabetes was using calcium supplementation. Thus, the baseline statistical differences seen in the group overall (men and women) were accounted for by male gender. Oral calcium supplementation dose subanalysis was inhibited by the small samples of patients taking various doses. All patients were using $>500 \mathrm{mg} /$ day and $<2000 \mathrm{mg} /$ day.

\section{Progression of AVC}

Overall Group (Including Men)

Overall aortic valve calcification progressed by $41 \pm 7.7 \mathrm{U}$ in the $3.7 \pm 0.9$ years of follow-up $(P<$ .001). Within this group, those who used calcium supplementation showed no difference in the rate 
Table 2. Baseline Characteristics in the Overall Female Population and in Subgroups of Females Both Taking and Not Taking Calcium Supplementation*

\begin{tabular}{|c|c|c|c|c|}
\hline Variable & $\begin{array}{c}\text { Overall } \\
(\mathrm{n}=144)\end{array}$ & $\begin{array}{l}\text { No Supplement } \\
\quad(\mathrm{n}=119)\end{array}$ & $\begin{array}{l}\text { Supplement } \\
\quad(\mathrm{n}=25)\end{array}$ & $P$ \\
\hline Age (yr) & $67.8 \pm 5.2$ & $67.6 \pm 5.0$ & $68.5 \pm 5.9$ & .42 \\
\hline Male Gender (n [\%]) & $0(0)$ & $0(0)$ & $0(0)$ & \\
\hline Body Mass Index $\left(\mathrm{kg} / \mathrm{m}^{2}\right)$ & $28.6 \pm 5.5$ & $28.8 \pm 5.7$ & $27.2 \pm 4.7$ & .17 \\
\hline Age $<70$ years $(\mathrm{n}[\%])$ & $97(67)$ & $83(70)$ & $14(56)$ & .18 \\
\hline Smoking (pack/yr) & $6.6 \pm 12.6$ & $6.6 \pm 12.4$ & $6.5 \pm 14.1$ & .94 \\
\hline History of HTN (n [\%]) & $86(60)$ & $74(62)$ & $12(48)$ & .19 \\
\hline Treated DM (n [\%]) & $11(8)$ & $10(8)$ & $1(4)$ & .45 \\
\hline Tchol (mg/dL) & $220.2 \pm 34.4$ & $219.2 \pm 35.2$ & $225 \pm 30.1$ & .44 \\
\hline LDL (mg/dL) & $124.8 \pm 30.7$ & $124.2 \pm 31.4$ & $127.8 \pm 27.3$ & .6 \\
\hline HDL (mg/dL) & $58.6 \pm 15.9$ & $58.3 \pm 16.4$ & $60.1 \pm 13.3$ & .61 \\
\hline $\mathrm{TG}(\mathrm{mg} / \mathrm{dL})$ & $185.5 \pm 80.0$ & $185.5 \pm 81.4$ & $185.8 \pm 74.6$ & .98 \\
\hline CAC score (base) & $160.7 \pm 383.5$ & $183.9 \pm 416.9$ & $50.4 \pm 79.3$ & .11 \\
\hline AVC score (base) & $29.8 \pm 91.0$ & $30.7 \pm 93.8$ & $25.4 \pm 77.9$ & .79 \\
\hline Serum calcium & $9.5 \pm 0.4$ & $9.5 \pm 0.4$ & $9.4 \pm 0.3$ & .38 \\
\hline GFR (MDRD) & $66.3 \pm 17.0$ & $65.3 \pm 18.3$ & $69.3 \pm 12.7$ & .34 \\
\hline
\end{tabular}

*Data source: the Epidemiology of Coronary Artery Calcification (ECAC) study. ${ }^{12}$

HTN, hypertension; DM, diabetes mellitus; LDL, low-density lipoprotein; HDL, high-density lipoprotein; TG, triglycerides; CAC, coronary artery calcification; AVC, aortic valve calcification; GFR, glomerular filtration rate; MDRD, Modification of Diet in Renal Disease equation.

of progression (40 $\pm 29 \mathrm{U}$ ) compared with those who did not take calcium supplements $(41.4 \pm 8.0$ $\mathrm{U})$ in the 3.7 year follow-up $(P<.96)$. In addition, there was no regression of aortic valve calcification or stenosis in either group or the group overall.

\section{Women Only}

The entire cohort of women showed a statistically significant increase in AVC score from $29.8 \pm 7.6$ at baseline to $62.8 \pm 15.6 \mathrm{U}$ at follow-up $(P<$ .0007). Of the 144 women included in this study, 25 reported using calcium supplementation at baseline. AVC in women using supplementation increased from $25 \pm 16 \mathrm{U}$ at baseline to $65 \pm 42 \mathrm{U}$ at follow-up $(P=.18)$. Those not using supplementation showed a significant increase in AVC: from $31 \pm 9$ at baseline to $62 \pm 17 \mathrm{U}$ at follow-up $(P<.002$; see Figure 1). The progressions over $3.7 \pm 0.9$ years were not significantly different between those taking supplementation $(39 \pm 28 \mathrm{U})$ and those not taking supplementation $(30 \pm 9 \mathrm{U}$; $P=.73$; see Figure 1). To correct for outlying data, we analyzed the log of the progression of AVC. We found that those women taking calcium supplementation had mean $\log$ values of $2.6 \pm 0.81 \mathrm{U}$ at baseline and $3 \pm 1.0 \mathrm{U}$ at follow-up, with a mean progression of $1.00 \pm 0.34 \mathrm{U}(P=.076)$ over $3.7 \pm$
0.9 years. The cohort not using supplements had mean $\log$ values of $2.9 \pm 0.25 \mathrm{U}$ at baseline and $3.5 \pm 0.25 \mathrm{U}$ at follow-up, with a mean progression

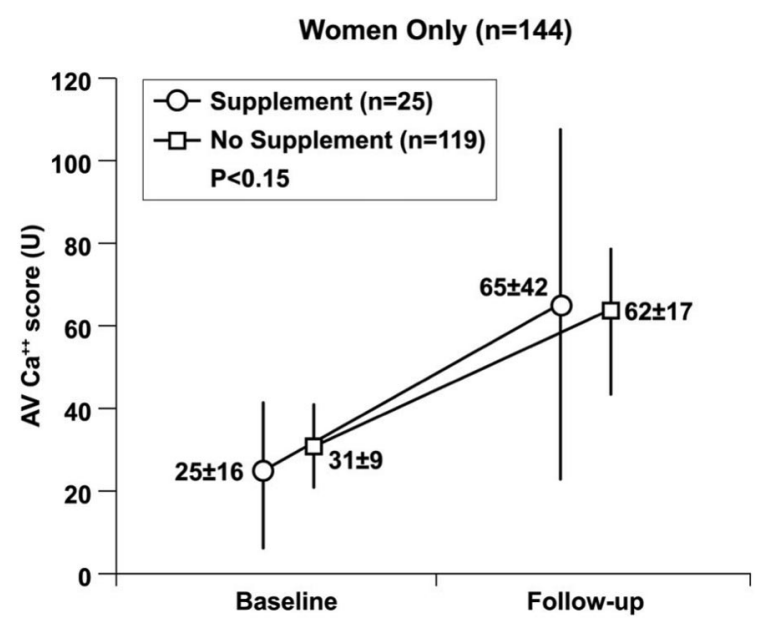

Figure 1. Comparison of the change in aortic valve calcification (AVC) from baseline to follow-up (vertical lines show SD) in women who took calcium supplementation and those that did not take supplementation. $P$ is comparing rates of change in AVC score (supplement vs no supplement). Data source: the Epidemiology of Coronary Artery Calcification (ECAC) study. ${ }^{12}$ 
of $1.00 \pm 0.11 \mathrm{U}(P<.001$ over $3.7 \pm 0.9$ years $)$. There was no significant difference in the progression between the 2 groups $(P=.91)$. A difference in AVC score of 85 between baseline and follow-up could be predicted with $80 \%$ power $(\alpha=0.05)$.

\section{Progression of CAC}

Overall Group (Including Men)

Of the 257 patients enrolled in the study overall, not all patients had follow-up CAC measurements. Only those patients with CAC scores both at baseline and follow-up were included in the analysis (203 patients not taking supplements; 25 patients taking supplements). Of the 203 patients not taking supplements, 108 were women. CAC progressed from $346 \pm 44 \mathrm{U}$ to $471 \pm 52 \mathrm{U}$ at follow-up in those not taking supplements, for mean progression value of $112 \pm 21 \mathrm{U}(P<.001)$ over $3.8 \pm 0.9$ years. Those using calcium supplementation progressed $50 \pm 16 \mathrm{U}$ to $97 \pm 28 \mathrm{U}$, for a mean progression of $46 \pm 15 \mathrm{U}(P<.004)$. The rates of progression were different $(P<.001)$ but the differences in sex and degree of baseline calcification necessitated assessment in women only.

\section{Women Only}

A comparison of the female cohort further delineated the effect of calcium supplementation on CAC. Those using supplementation had a mean CAC change from $50 \pm 16 \mathrm{U}$ to $97 \pm 28 \mathrm{U}$ at follow-up, resulting in a mean change of $47 \pm 15 \mathrm{U}$ $(P<.004)$ during a follow-up period of $3.7 \pm 0.9$ years. Patients not taking calcium supplementation had a baseline CAC of $184 \pm 32 \mathrm{U}$ with follow-up of CAC $275 \pm 54 \mathrm{U}$; a mean progression score of $112 \pm 22(P<.001)$. The difference in the mean changes between the 2 groups was not statistically significant $(P<.15$; see Figure 2$)$. Similar to the AVC analysis, we calculated progression rates using $\log$ values to account for outlying data. Among patients using calcium supplementation, the log CAC at baseline was $3.8 \pm 0.42 \mathrm{U}$ and at follow-up was $3.8 \pm 0.43 \mathrm{U}$; mean $\log$ progression $0.6 \pm 0.24$ $\mathrm{U}(P<.0006)$. In those subsets not taking supplements, the $\log$ CAC baseline was $4.2 \pm 0.2 \mathrm{U}$ and was at follow-up $4.6 \pm 0.20 \mathrm{U}$ (progression of $0.98 \pm 0.11 \mathrm{U} ; P<.001$ ). The rates of progression were not significantly different between groups $(P=.72)$. A minimal difference between cohorts in baseline to follow-up CAC score of 237 could be done with $80 \%$ power $(\alpha=0.05)$.

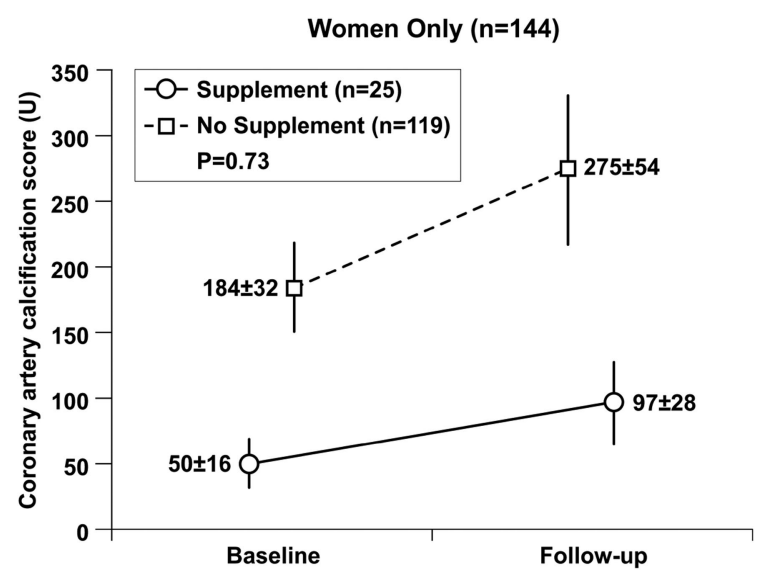

Figure 2. Comparison of the change in coronary artery calcification (CAC) from baseline to follow-up (vertical lines show SD) in women who took calcium supplementation and those that did not take supplementation. $P$ is comparing rates of change in coronary artery calcium score (supplement vs no supplement). Data source: the Epidemiology of Coronary Artery Calcification (ECAC) study. ${ }^{12}$

\section{Discussion}

This retrospective analysis of prospectively collected data shows that there is neither an increase nor a decrease in the rate of progression of either AVC or CAC in patients using supplemental calcium. This finding is important given that the use of oral calcium has been clearly shown to decrease bone degradation, potentially leading to stabilization in bone density and decreased vertebral fractures. ${ }^{16}$ These effects on bone metabolism have led to an overall increased use of calcium supplementation in the elderly populationthe same population that is most susceptible to AVC, aortic stenosis, and CAC.

Recently our understanding of the pathophysiology of AVC and CAC has expanded, raising clinical concerns that calcium supplementation may promote AVC and vascular calcification. These concerns are supported in part by evidence of upregulation of bone morphogenetic protein associated with valvular calcification, ${ }^{10}$ a process mimicking bone formation. Furthermore, in the first population-based study to objectively follow the natural progression of AVC, Messika-Zeitoun et $\mathrm{al}^{7}$ found that specific atherosclerotic risk factors accelerated the rate of progression of AVC. They proposed that the pathophysiology of AVC is characterized by 2 distinct phases. The first, or early, phase is characteristic of atherosclerosis and similar 
to the pathology of the early aortic valve lesion comprising inflammation, oxidized lipoprotein deposition, and colocalization with early calcium deposition. ${ }^{17}$ The later phase is characterized primarily by calcium ossification ${ }^{18}$ occurring at a rate correlating with calcification load, manifest by the CAC score ${ }^{19}$ but independent of atherosclerotic risk factors responsible for the early phase. Supporting their hypothesis, Mohler et $\mathrm{al}^{18}$ also found that AVC progresses independently of lipid levels and is unaffected by statin therapy, presumably because treatment was instituted during the late phase of AVC. Furthermore, the impact of calcium deposition is aggravated by the calcium load: the higher the calcium load the faster the calcium accumulates. ${ }^{7}$ Similarly, patients with aortic stenosis who have a higher calcium load experience clinical cardiac events at a higher rate than those with a lesser calcium load irrespective of the hemodynamic severity. ${ }^{20-22}$ Thus, based on this evidence, it could be anticipated that any intervention that either increases or reduces aortic calcium load may have important clinical implications. We were unable to draw conclusions based on oral calcium doses secondary to the small samples of patients taking the various doses. Reassuringly, however, our study found that the presence of oral calcium supplementation was associated with neither accelerated AVC nor CAC progression. The lower CAC calcium scores overall in the calcium supplement group were felt to be secondary to the nonrandomized nature of this study.

Hsai et $\mathrm{al}^{23}$ recently published a study of 36,282 female patients randomized to calcium supplementation or placebo and found no difference in the natural outcome rates of coronary events or stroke. This result, combined with our finding of no variation in the rate of progression of AVC or CAC, supports the evidence that calcium supplementation is not associated with an increase in adverse cardiovascular effects.

The Women's Health Initiative database provided insight with objective measurement of coronary artery calcium scores and their natural progression. ${ }^{24}$ Similar to the natural progression of AVCs, the baseline calcium scores correlated directly with progression rate. The database, however, did not attempt to correlate these scores with oral calcium supplementation use or objective measurement of AVC with EBCT. This is the first study to look at AVC and CAC in the context of calcium supplementation.

\section{Limitations}

This study was based on data collected from the ECAC $^{12,25}$ database and AVC substudy. ${ }^{7}$ Thus, because this was not a clinical trial we cannot rule out a beneficial or deleterious effect of a therapeutic intervention. The small sample size of 25 patients also makes it difficult to draw significant conclusions. The minimal difference in the AVCs and CACs that could be detected by our sample size was found to be 85 and 237, respectively, with $80 \%$ power and $\alpha$ of 0.05 . A rate of progression of $95 \pm$ $80 \mathrm{AU} / \mathrm{year}$ was found to have clinical significance with hemodynamic changes in the previously published report about this database. ${ }^{7}$ Our prospective study was powered to measure changes of $\geq 28$ AU/year (85 during a 3 -year follow-up period). Progression that occurs at less than this rate is felt to be clinically insignificant based on data from our previous study. The small size of the cohort potentially could mask smaller increases in the rate of progression that may become evident after a longer follow-up, but this would need to be assessed using a much larger database with a substantially longer follow-up time. The use of EBCT to objectively measure calcium deposition on the aortic valve and coronary arteries is a more sensitive marker of valvular calcification than echocardiogram, ${ }^{26}$ also adding to the power of the study.

The additional disadvantage to the small sample size was the inability to detect differences in the rate of progression associated with higher intake of oral calcium or with different disease states, such as diabetes. In addition, the overall baseline CAC scores and HDL levels were statistically significant in the groups; however, when only the female cohorts were compared, neither of these reached significance $(P=.11)$. With only 25 patients it would not be possible to differentiate characteristics that may have led to this discrepancy in the overall group, but may be related to sex alone. These types of analyses would necessitate a much larger cohort with more detailed history taking, with emphasis on dietary intake of calcium in addition to oral calcium supplementation.

\section{Conclusion}

The prevalence of AVC and CAC is increasing as our population ages. The use of oral calcium sup- 
plementation is also increasing among this same demographic. In this small study with 4-year follow-up, we found that calcium supplementation use in a general community population did not affect the progression of AVC and CAC. Larger prospective, randomized studies are needed to confirm these findings.

\section{References}

1. Pomerance A. Ageing changes in human heart valves. Br Heart J 1967;29:222-31.

2. American Association of Clinical Endocrinologists Osteoporosis Task Force. American Association of Clinical Endocrinologists 2001 Medical Guidelines for Clinical Practice for the Prevention and Management of Postmenopausal Osteoporosis. Endocr Pract 2001;7:293-312.

3. Heinemann DF. Osteoporosis. An overview of the National Osteoporosis Foundation clinical practice guide. Geriatrics 2000;55:31-6.

4. US Department of Health and Human Services, Office of the Surgeon General. Bone health and osteoporosis: a report of the Surgeon General. Rockville, MD: US Department of Health and Human Services; 2004.

5. Iung B, Baron G, Butchart EG, et al. A prospective survey of patients with valvular heart disease in Europe: the Euro Heart Survey on Valvular Heart Disease. Eur Heart J 2003;24:1231-43.

6. Nkomo VT, Gardin JM, Skelton TN, Gottdiener JS, Scott CG, Enriquez-Sarano M. Burden of valvular heart diseases: a population-based study. Lancet 2006;368:1005-11.

7. Messika-Zeitoun D, Bielak LF, Peyser PA, et al. Aortic valve calcification: determinants and progression in the population. Arterioscler Thromb Vasc Biol 2007;27:642-8.

8. Hurle JM, Colvee E, Fernandez-Teran MA. The surface anatomy of the human aortic valve as revealed by scanning electron microscopy. Anat Embryol (Berl) 1985;172:61-7.

9. Tedgui A, Mallat Z. Anti-inflammatory mechanisms in the vascular wall. Circ Res 2001;88:877-87.

10. Mohler ER 3rd, Chawla MK, Chang AW, et al. Identification and characterization of calcifying valve cells from human and canine aortic valves. J Heart Valve Dis 1999;8:254-60.

11. Caira FC, Stock SR, Gleason TG, et al. Human degenerative valve disease is associated with up-regulation of low-density lipoprotein receptor-related protein 5 receptor-mediated bone formation. J Am Coll Cardiol 2006;47:1707-12.

12. Bielak LF, Sheedy PF 2nd, Peyser PA. Coronary artery calcification measured at electron-beam CT: agreement in dual scan runs and change over time. Radiology 2001;218:224-9.

13. Levey AS, Bosch JP, Lewis JB, Greene T, Rogers N, Roth D. A more accurate method to estimate glomerular filtration rate from serum creatinine: a new prediction equation. Modification of Diet in Renal Disease Study Group. Ann Intern Med 1999;130: 461-70.

14. Maher JE, Raz JA, Bielak LF, Sheedy PF 2nd, Schwartz RS, Peyser PA. Potential of quantity of coronary artery calcification to identify new risk factors for asymptomatic atherosclerosis. Am J Epidemiol 1996;144:943-53.

15. Robb RA. The biomedical imaging resource at Mayo Clinic. IEEE Trans Med Imaging 2001;20:854-67.

16. Shea B, Adachi A, Cranney A, et al. Calcium supplementation on bone loss in postmenopausal women. Cochrane Database Syst Rev 2004;(1):CD004526.

17. Olsson M, Thyberg J, Nilsson J. Presence of oxidized low density lipoprotein in nonrheumatic stenotic aortic valves. Arterioscler Thromb Vasc Biol 1999;19:1218-22.

18. Mohler ER 3rd, Gannon F, Reynolds C, Zimmerman R, Keane MG, Kaplan FS. Bone formation and inflammation in cardiac valves. Circulation 2001; 103:1522-8.

19. Yoon HC, Emerick AM, Hill JA, Gjertson DW, Goldin JG. Calcium begets calcium: progression of coronary artery calcification in asymptomatic subjects. Radiology 2002;224:236-41.

20. Messika-Zeitoun D, Aubry MC, Detaint D, et al. Evaluation and clinical implications of aortic valve calcification measured by electron-beam computed tomography. Circulation 2004;110:356-62.

21. Rosenhek R, Binder T, Porenta G, et al. Predictors of outcome in severe, asymptomatic aortic stenosis. N Engl J Med 2000;343:611-7.

22. Rosenhek R, Klaar U, Schemper M, et al. Mild and moderate aortic stenosis. Natural history and risk stratification by echocardiography. Eur Heart J 2004;25:199-205.

23. Hsia J, Heiss G, Ren H, et al. Calcium/vitamin D supplementation and cardiovascular events. Circulation 2007;115:846-54.

24. Hsia J, Klouj A, Prasad A, Burt J, Adams-Campbell LL, Howard BV. Progression of coronary calcification in healthy postmenopausal women. BMC Cardiovasc Disord 2004;4:21.

25. Peyser PA, Bielak LF, Chu JS, et al. Heritability of coronary artery calcium quantity measured by electron beam computed tomography in asymptomatic adults. Circulation 2002;106:304-8.

26. Morgan-Hughes GJ, Roobottom CA, Marshall AJ. Aortic valve imaging with computed tomography: a review. J Heart Valve Dis 2002;11:604-11. 